\title{
Simultaneous consumption of pentose and hexose sugars: an optimal microbial phenotype for efficient fermentation of lignocellulosic biomass
}

\author{
Jae-Han Kim • David E. Block • David A. Mills
}

Received: 9 July 2010 /Revised: 9 August 2010 /Accepted: 11 August 2010 /Published online: 14 September 2010

(C) The Author(s) 2010. This article is published with open access at Springerlink.com

\begin{abstract}
Lignocellulosic biomass is an attractive carbon source for bio-based fuel and chemical production; however, its compositional heterogeneity hinders its commercial use. Since most microbes possess carbon catabolite repression (CCR), mixed sugars derived from the lignocellulose are consumed sequentially, reducing the efficacy of the overall process. To overcome this barrier, microbes that exhibit the simultaneous consumption of mixed sugars have been isolated and/or developed and evaluated for the lignocellulosic biomass utilization. Specific strains of Escherichia coli, Saccharomyces cerevisiae, and Zymomonas mobilis have been engineered for simultaneous glucose and xylose utilization via mutagenesis or introduction of a xylose metabolic pathway. Other microbes, such as Lactobacillus brevis, Lactobacillus buchneri, and Candida shehatae possess a relaxed CCR mechanism, showing simultaneous consumption of glucose and xylose. By exploiting CCR-negative phenotypes, various integrated processes have been developed that incorporate both enzyme hydrolysis of lignocellulosic material and mixed sugar
\end{abstract}

J.-H. Kim • D. E. Block • D. A. Mills $(\bowtie)$

Robert Mondavi Institute for Wine and Food Science,

Department of Viticulture and Enology, University of California,

One Shields Avenue,

Davis, CA 95616, USA

e-mail: damills@ucdavis.edu

\section{E. Block}

Department of Chemical Engineering and Materials Science, University of California,

One Shields Avenue,

Davis, CA 95616, USA fermentation, thereby enabling greater productivity and fermentation efficacy.

Keyword Lactobacillus brevis · Carbon catabolite repression - Simultaneous carbohydrate utilization . Lignocellulosic biomass $\cdot$ SSMSF

\section{Introduction}

Use of lignocellulosic biomass is currently under intensive study as an alternative growth substrate for bio-based chemical and energy production. Despite the advantages in sustainability and availability, commercial use of lignocellulose is still problematic. Due to the complexity of lignocellulosic materials, hydrolysis of hemicellulose and cellulose into five- and six-carbon sugars has to be carried out prior to, or concurrently with, the fermentation. During the fermentation of sugars released by hydrolysis, microorganisms tend to selectively utilize a preferred sugar, usually glucose. This preferential consumption of sugar, termed carbon catabolite repression (CCR), makes it challenging to design and efficiently control the fermentation processes using lignocellulosic biomass as a feedstock.

The development of microorganisms to ferment sugars released from lignocellulosic biomass, either through selection of new strains or by genetic engineering of traditional strains, has generally focused on generating maximum product yield from total available sugar. However, the pattern of substrate utilization is also important in overall process design as simultaneous use of all sugars is likely to lead to a shorter and more productive process. In this review, we present several microbial strains that can utilize mixed sugars simultaneously and discuss the 
advantages in lignocellulosic biomass utilization in terms of fermentation process design.

\section{Fermentation of mixed sugars derived from lignocellulosic biomass}

Lignocellulose is one of the structural materials of the plant cell wall that contains the heterogeneous complex of cellulose, hemicellulose, and lignin (Buchanan et al. 2000). Cellulose is a $\beta$-1,4-linked homopolymer of glucose, whereas hemicellulose is a heteropolymer of hexoses (glucose) and pentoses (mainly xylose and arabinose). Lignin, another component of lignocellulose, is a heterogeneous polymer of phenylpropanoid units that gives the tensile strength of plant material (Boerjan et al. 2003). To use a lignocellulosic biomass as a substrate for a biobased chemical production, hydrolysis of cellulose and hemicellulose has to be achieved prior to the fermentation of the resulting mixed sugars. Hemicellulose can be chemically degraded at high pressure and high temperature by chemicals such as ammonia (Ammonia fiber explosion: AFEX) or at ambient temperature by dilute sulfuric acid $(<1.2 \%)$ (Dale and Moreira 1982; Holtzapple et al. 1992; Ropars et al. 1992; Schell et al. 1991). However, cellulose fiber is hydrolyzed to glucose enzymatically by the commercially available cellulases and cellobiases.

Lignocellulosic biomass is comprised of up to $45 \%$ hemicellulose. Therefore, in order to achieve maximum product yield and productivity, a complete utilization of mixed sugars derived from hemicellulose is essential (Saha 2003). While many fermentation microbes are able to utilize pentose sugars, catabolism of these pentose sugars is typically suppressed by glucose derived from cellulose (Stulke and Hillen 1999). This selective and sequential utilization of mixed sugars by most microbes makes the fermentation process complex and often reduces the yields and productivity on the target biomass (Bothast et al. 1999). When sugars are consumed sequentially during the fedbatch fermentations, the non-preferred sugars (such as pentoses) accumulate in the media until the preferred sugar (i.e., glucose) is completely consumed (Fig. 1a). Due to the high concentration of inhibitory fermentative end products, such as lactic acid or ethanol, the utilization rate of the remaining pentose sugars is slower than if it had been fermented as a sole carbohydrate. Furthermore, if a small amount of glucose is left in the media, pentoses will not be utilized and remain unfermented (Fig. 1b). To avoid the accumulation of pentoses, sequential fermentations of all available glucose and pentose sugars have to be repeated after addition of new substrates during fed-batch fermentation (Fig. 1c). This frequent change of available carbon sources causes a non-steady state of growth, making it
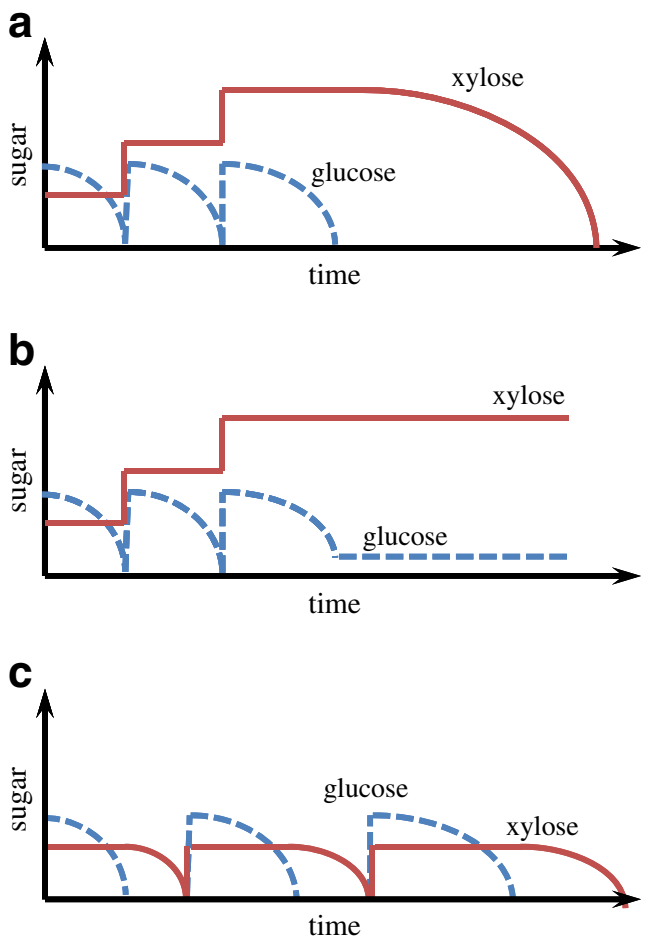

Fig. 1 Schematic diagram of mixed sugar utilization during fed-batch fermentation by CCR positive strain. a Pentoses (line) accumulate while glucose (dotted line) is completely consumed. Remaining pentoses is utilized only after depletion of glucose. b Pentoses (line) accumulate during the consumption of glucose. Due to the incomplete consumption of glucose, pentoses cannot be fermented. c Repetitive sequential utilization of glucose and pentoses. Additional mixed sugar substrate is added after complete and sequential consumption of glucose and pentoses

difficult to predict and control the fermentation process and causing delays between different growth phases. Sequential utilization of mixed sugars, therefore, limits the overall process design and optimization, and restricts maximum yield and productivity of lignocellulosic biomass.

An integrated process of hydrolysis and fermentation, termed simultaneous saccharification and fermentation (SSF), has been well established using cellulosic fiber as a sole carbon source. The advantage of SSF is the removal of glucose by fermentation preventing feedback inhibition of the cellulolytic enzyme. However, to ensure that no glucose remains in the medium, the glucose utilization rate of the microorganism needs to be higher than the glucose production rate by enzymatic hydrolysis. In other words, maximum efficacy of SSF can be achieved when the fermentative microbes are maintained at the interface between substrate-limiting and substrate-rich conditions. During SSF, the fermentative microbe constantly consumes glucose which, in turn, activates CCR. However, if lignocellulosic biomass is used as a substrate, then the overall SSF process must contend with sequential mixed substrate utilization. Pentose sugars will accumulate and remain unfermented in the media during the SSF process. If 
carried out in a fed-batch mode, sequential utilization by the host microorganism results in a consecutive repeat of SSF and pentose fermentation which does not truly achieve a full integration of hydrolysis and fermentation.

One solution to the problem of sequential utilization of lignocellulosic-derived sugars is to employ CCR-negative microorganisms. With such microbes, pentoses are coutilized with glucose and accumulation of pentose sugars did not occur during the fermentation. If pentose utilization is as efficient as glucose utilization, the fermentation process can be designed as if a single carbohydrate were present in the medium. CCR-negative strains have particular merit when operating in SSF mode using lignocellulose hydrolysate (termed simultaneous saccharification and co-fermentation; SSCF). In such a process, pentose utilization and SSF of cellulosic fiber occur independently in a single reactor without the interference from each process. In SSCF, microbial utilization of glucose maximizes the cellulolytic activity by removing feedback inhibition. At the same time, the simultaneous pentose metabolism ensures that microorganisms maintain optimum and steady state cellular activity.

\section{Bacterial carbon catabolite repression}

Microorganisms regulate their catabolic activity in response to environmental nutrients. Because glucose is the most abundant carbohydrate monomer, bacterial metabolic pathways have evolved for glucose utilization and, therefore, efficient generation of energy is achieved through oxidation of glucose (Lengeler et al. 1999). At the same time, most bacteria possess alternative sugar utilization mechanisms as well to survive in competitive mixed sugar environments. In this case, hierarchical control of sugar utilization is accomplished by a complex regulatory mechanism and is a common phenomenon of bacteria (Lengeler et al. 1999). Although the control mechanisms, often called CCR, are different between Gram-negative and Gram-positive bacteria, the use of a secondary sugar is generally controlled in two layers. In the first layer, the transport of a secondary sugar is inhibited when a preferred sugar is present, a process called inducer exclusion (or repulsion). In the second layer, transcription of catabolic enzyme for a secondary sugar is regulated by two mechanisms. First, the expression of catabolic enzymes requires an inducer. Commonly, transcription of catabolic enzymes is suppressed by a repressor protein that is released by binding with the secondary sugar. Second, transcription of catabolic enzymes is co-repressed by a carbon catabolite control protein $(\mathrm{CcpA}$; in Gram-positive bacteria (Stulke and Hillen 2000)) or activated by catabolite repression protein (CRP) complex (in Gram-negative bacteria (Stulke and Hillen 1999)).

\section{Co-consumption of mixed sugars by CCR-negative strains}

Targeted genetic engineering for a CCR-negative phenotype

Escherichia coli is widely used as a host strain for industrial processes. As described above, three different mechanisms are involved in CCR in E. coli. Inducer exclusion is achieved by being coupled to the phosphoenolpyruvate-dependent sugar phosphotransferase system (PTS) for each specific carbohydrate (Lengeler 1996). As shown in Fig. 2a, translocation occurs by the specific sugar transporter of the EII B and EII C complex (EII BC) accompanied by the phosphorylation of the respective sugar. The phosphate group originates on phosphoenolpyruvate (PEP) and is transferred sequentially to EI, HPr, and finally to EII A. Among these components, the glucose-specific EII A (EII $\mathrm{A}^{\text {glc }}$ ) is a key element for CCR in E. coli. In the presence of glucose, dephosphorylated EII $\mathrm{A}^{\text {glc }}$ is more abundant than phosphorylated EII A (EII $\mathrm{A}^{\text {glc }}-P$ ) because the phosphate group in EII $\mathrm{A}$ is transferred to glucose with high affinity. EII $A^{\text {glc }}$ then binds onto the EII BC complex of carbohydrates other than glucose and inhibits translocation of these other sugars (a process called inducer exclusion). When glucose is absent, EII A ${ }^{\text {glc }}$ - $P$ predominates and cannot bind the EII BC complexes of other carbohydrates, which relives the inducer exclusion. In addition to inducer exclusion, EII $\mathrm{A}^{\text {glc }}$ plays an important role in CRP-cAMP-mediated activation of catabolic gene transcription (Fig. 2b). Phosphorylated EII $A^{\text {glc }}\left(E^{2} A^{\text {glc }}-P\right.$ ) activates the adenyl cyclase (AC), which, in turn, converts ATP to cyclic AMP (cAMP). The CRP then binds with cAMP and interacts with the upstream regulation region of catabolic operons for alternative sugars, thereby activating transcription.

A CCR-negative phenotype of $E$. coli has been achieved by the manipulation of the $p s t G$ gene encoding the EII $\mathrm{A}^{\mathrm{glc}}$. Through a deletion mutation of $p t s G$, inducer exclusion can be relieved enabling the translocation of alternative sugars in the presence of glucose. As noted, EII $\mathrm{A}^{\text {glc }}$ is also involved in the CRP-cAMP-mediated transcriptional activation of catabolic genes for the alternative sugar. However, disruption of pts $G$ successfully achieved simultaneous sugar consumption suggesting that the inducer exclusion is more important for CCR (Kimata et al. 1997). Based on this observation, Nichols and co-workers engineered an $E$. coli strain that is a CCR-negative phenotype and produce ethanol exclusively from lignocellulosic materials (Nichols 
a

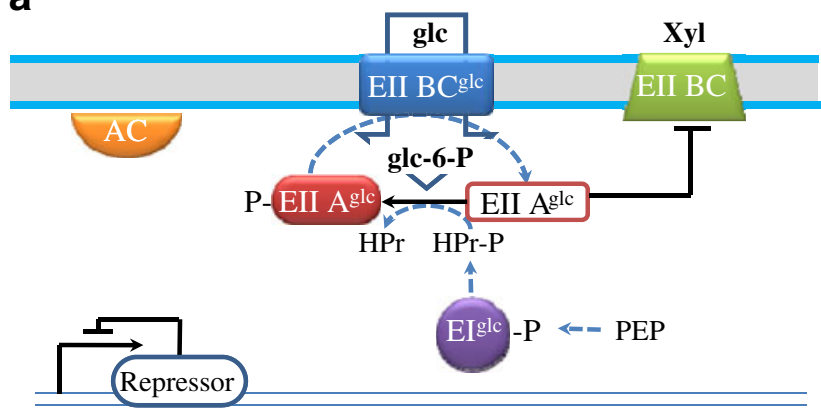

b

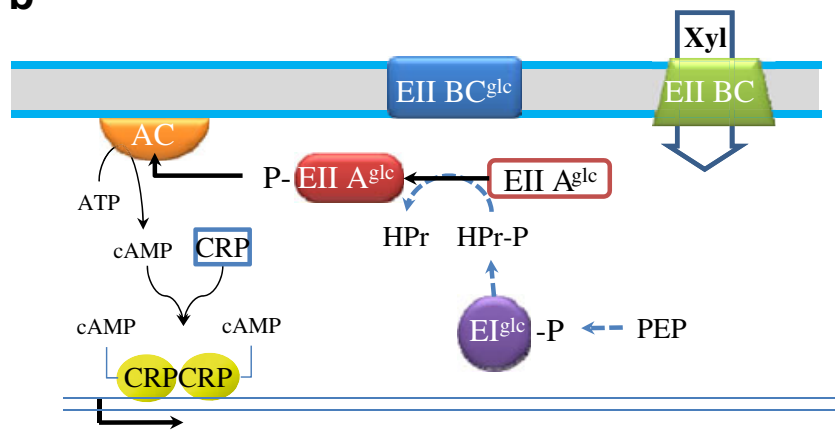

C

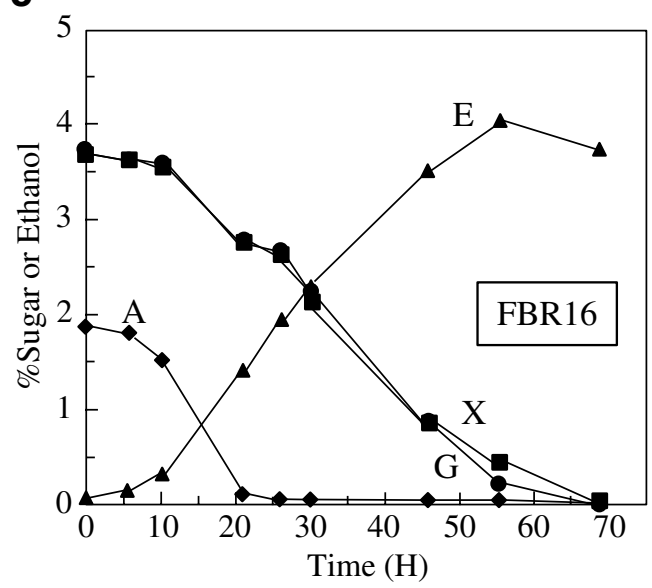

Fig. 2 Carbon catabolite repression of E. coli and simultaneous sugar utilization by CCR-negative strain. a Schematic diagram of inducer exclusion and $\mathbf{b}$ CRP mediated transcription activation in E. coli. c Simultaneous sugar utilization profile by $p t s G$ knockout mutant of $E$. coli FRB16 (Nichols et al. 2001). Symbols: (filled circle) glucose; (filled square), xylose; (filled diamond), arabinose; (filled triangle), ethanol. Abbreviations: $A C$, adenylate cyclase; glc, glucose; glc-6- $P$, glucose 6-phosphate; PEP, phosphoenolpyruvate; $x y l$, xylose; $E I I A^{g l c}$, EII A component of glucose-specific PTS; EII BC $C^{g l c}$, EII B and EII C components of glucose-specific PTS; $E I^{\text {glc }}$, EI component of glucosespecific PTS; CRP, catabolite repression protein

et al. 2001). Starting from E. coli NZN111, a strain that contains knockout mutations in lactate dehydrogenase $(l d h A)$ and pyruvate-formate lyase ( $p f l$ ) genes (Bunch et al. 1997), Nichols and co-workers further deleted the fumarate dehydrogenase $(\triangle \mathrm{frdABCD})$, preventing the pro- duction of succinic acid to minimize byproduct formation. For production of ethanol, a plasmid encoding both pyruvate decarboxylase $(p d c)$ and alcohol dehydrogenase $(a d h A)$ was introduced to construct an ethanol-producing pathway. At the same time, the CCR-negative variant was generated by knocking out the pts $G$ gene. As a result, xylose and arabinose were simultaneously consumed as fast as glucose, suggesting the catabolic pathways for xylose and arabinose were fully activated despite the presence of glucose in the medium (E. coli FRB16; Fig. 2c).

Co-utilization of mixed sugars by metabolic pathway implantation

In addition to the modulation of a specific CCR component, different metabolic engineering approaches result in a simultaneous sugar utilization in microorganisms that do not naturally use five-carbon sugars, even in the absence of glucose. To enable pentose utilization, the metabolic pathway for xylose and/or arabinose metabolism of $E$. coli was transferred into Zymomonas mobilis (Lawford and Rousseau 2002; Zhang et al. 1995). Since the pentose metabolic pathway came from an exogenous source, $Z$. mobilis host regulation (CCR or otherwise) did not govern its expression, resulting in co-utilization of glucose and pentoses (Joachimsthal and Rogers 2000; Lawford and Rousseau 2002). However, since Z. mobilis did not have a specific transporter for xylose (or arabinose) (Parker et al. 1995), the consumption of pentoses was slow. When this strain was grown on the same initial amounts of xylose and glucose, less than $10 \%$ of the xylose was consumed, while the glucose was completely depleted. Perhaps future engineering to introduce an exogenous pentose transport system into this organism will further increase pentose coutilization in this strain.

Saccharomyces cerevisiae has long history of development as a host strain for ethanol production from renewable resources, including lignocellulosic biomass. The genes necessary for xylose catabolism have been incorporated into $S$. cerevisiae to produce ethanol from mixtures of glucose and xylose (Hahn-Hagerdal et al. 2007; Ho et al. 1998; Kuyper et al. 2004; Sedlak and Ho 2004a). As was the case with Z. mobilis, heterologous xylose catabolism was expressed regardless of the presence of glucose, thus enabling the simultaneous utilization of glucose and xylose. In spite of this capacity, a lack of xylose-specific transporter in engineered strains has hindered the efficient use of xylose in a mixed sugar fermentation. It has been reported that $S$. cerevisiae transports xylose and glucose using the same transporter, and the presence of glucose inhibited xylose uptake and its utilization rate (Meinander and HahnHagerdal 1997; Sedlak and Ho 2004b). While S. cerevisiae has 18 different hexose transport proteins 
(Leandro et al. 2009), a study of glucose and xylose uptake kinetics revealed that $S$. cerevisiae exhibited low and high affinity uptake systems, respectively, depending on the extracellular concentration of glucose. Xylose could be transported both cases; however, the transporters exhibited a low affinity for xylose when the glucose concentration was high. Conversely, when the extracellular glucose concentration was low, there was an $85 \%$ increase in the maximum xylose transport rate (Lee et al. 2002). Therefore, in order to fully enable the co-utilization of xylose and glucose in this organism, glucose had to be maintained at a low concentration (Meinander et al. 1999; Pitkanen et al. 2003; Lee et al. 2003). Like the engineered Z. mobilis strain, the amount of xylose consumed was only $10-20 \%$ of glucose in mixed sugar fermentation. Moreover, the xylose utilization rate was still slow even after depletion of glucose since xylose alone cannot match the redox balance of the cell (Lau and Dale 2009; Sedlak and Ho 2004b). To enable the effective co-utilization of xylose and glucose during SSCF, Olofsson and co-workers suggested modulation of the cellulase level to maintain a low (but not zero) concentration of glucose in order to promote the xylose uptake and metabolism. In this fashion, total xylose consumption was increased to $40-80 \%$ (Bertilsson et al. 2009; Olofsson et al. 2010).

CCR-negative lactic acid bacteria for lignocellulosic biomass utilization

CCR for lactic acid bacteria (LAB) has been reported in Lactobacillus casei (Gosalbes et al. 1999; Veyrat et al. 1994; Viana et al. 2000), Lactobacillus pentosus (Chaillou et al. 2001; Chaillou et al. 1999; Mahr et al. 2000), Lactobacillus plantarum (Marasco et al. 1998), Lactobacillus sakei (Zuniga et al. 1998), and Lactobacillus delbrueckii (Morel et al. 1999; Schick et al. 1999). The regulatory mechanism for sugar utilization in LAB is similar to that in Bacillus subtilis, which possesses CcpAmediated and PTS-related CCR mechanisms (Stulke and Hillen 1999; Titgemeyer and Hillen 2002; Warner and Lolkema 2003). As summarized in Fig. 3a, inducer exclusion is coupled with PTS. However, instead of EII $\mathrm{A}$, the histidine-containing phosphocarrier protein $\mathrm{HPr}$ plays a key role in CCR. Histidine-phosphorylated Hpr (P-[His]Hpr) transfers a phosphate group to incoming carbohydrate via the EII BC complex; however, $\mathrm{P}$-[His] $\mathrm{Hpr}$ then interacts with the EII BC complexes of other carbohydrates to prevent the translocation of alternative sugars as well. At the same time, some Hpr is involved in a CcpA-mediated transcription repression. Upon activation by glycolytic metabolites such as a fructose 1, 6 bisphosphate (Fru-1,6-Bis $P$ ), another enzyme, Hpr kinase, phosphorylates the serine residue of $\mathrm{Hpr}$ (P-[Ser]Hpr). P-[Ser]Hpr then complexes with CcpA and Fru-1,6-Bis $P$. A complex of CcpA/P-[Ser]Hpr/Fru-1,6-Bis $P$ lands on the catabolite repression element (cre) sequence in the up-
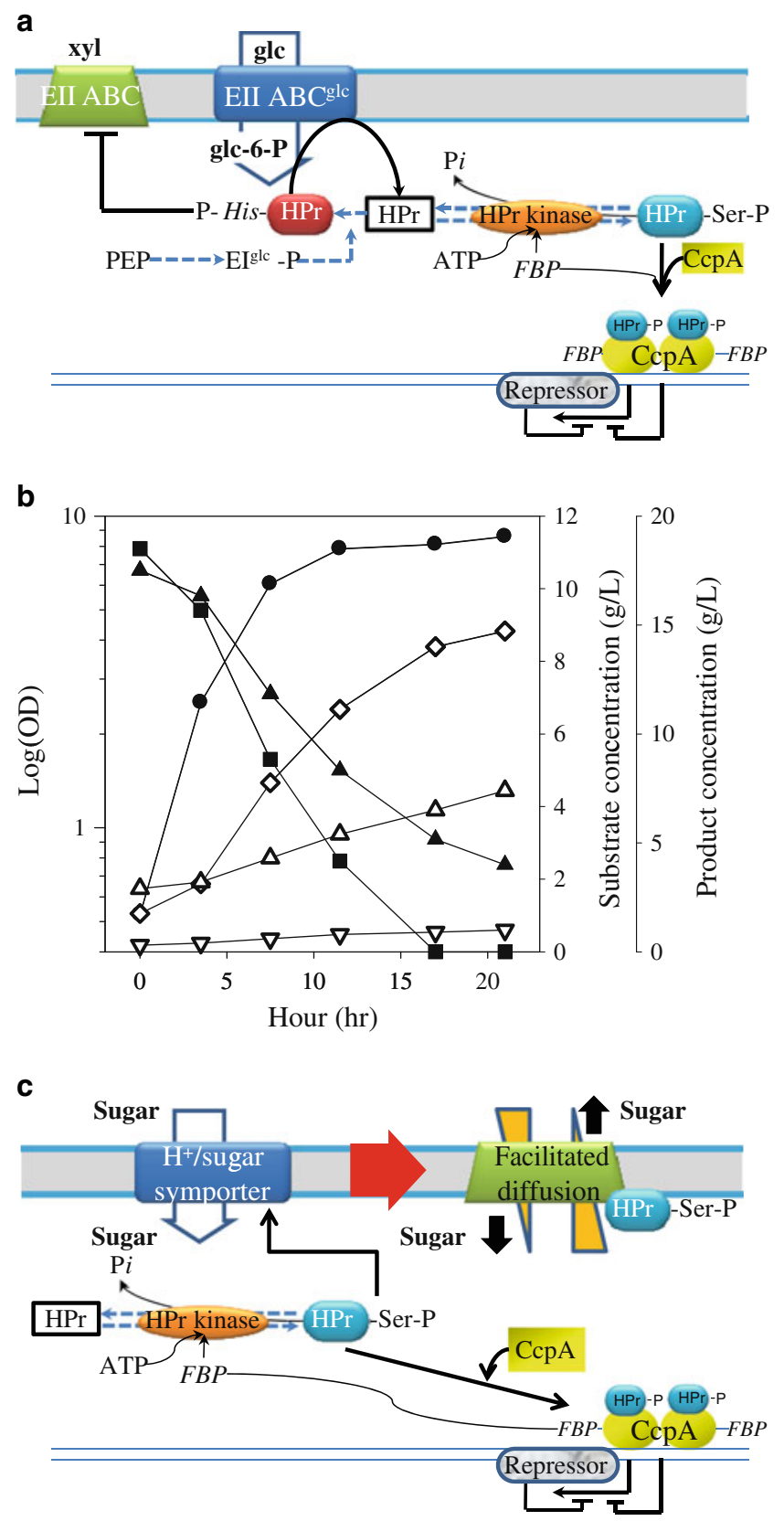

Fig. 3 Carbon catabolite repression of LAB and simultaneous sugar utilization by $L$. brevis. a Schematic diagram of carbon catabolite repression in LAB. b Simultaneous glucose and xylose utilization by L. brevis ATCC14869 (the figure was recreated from data presented in Kim et al. 2009). Symbols: (filled circle), glucose; (filled square), xylose; (empty triangle), acetate; (inverted triangle), ethanol; (empty diamond), lactate. $\mathbf{c}$ Relaxed mode of carbon catabolite repression in $L$. brevis. Abbreviations: glc, glucose; glc-6- $P$, glucose 6-phosphate; $P E P$, phosphoenolpyruvate; FBP, fructose bisphosphate; xyl, xylose; EII $A B C^{g l c}$, EII A, EII B, and EII C components of glucose-specific PTS; CсpA, carbon catabolite protein $\mathrm{A}$ 
stream region of catabolic operons and inhibits transcription initiation.

Recently, we reported that Lactobacillus brevis, a facultative heterofermentative $\mathrm{LAB}$, is able to simultaneously utilize any fermentable sugars present in the media (Kim et al. 2009). As shown in Fig. 3b, both glucose and xylose were consumed simultaneously and completely. Importantly, xylose was utilized as efficiently as glucose exhibiting similar specific consumption rates. Moreover, the specific consumption rate of total sugar during the mixed sugar fermentation was the same as when either glucose or xylose was fermented separately as a sole carbon source. These fermentation kinetics enable a mixed sugar fermentation process to be designed as if a single carbon source existed in the medium. When rice straw hydrolysate was used as a carbon source, the resulting mixed sugars were utilized simultaneously as well, and the kinetic parameters were equivalent to mixed sugar fermentations using rich media. These results suggest that $L$. brevis is also resistant to possible inhibitors commonly present in lignocellulosic hydrolysate. When applied in an integrated process (termed simultaneous saccharification and mixed sugar fermentation (SSMSF)), the hydrolysis of cellulose and fermentation of mixed sugars were carried out independently, yet simultaneously, without cross inhibition. Efficient consumption of glucose by L. brevis completely relieved feedback inhibition of cellulase and cellobiase. Moreover, xylose and arabinose derived from hemicellulose were simultaneously consumed, thus providing a consistent energy source even when concentration of glucose reached zero to remove the feedback inhibition of enzymes. As a consequence, sugar utilization and product formation profiles during SSMSF were similar to those observed in a control mixed sugar fermentations using rich media (Kim et al. 2010b).

While components of CCR have been observed in $L$. brevis (Chaillou et al. 1998; Kim et al. 2009; Reizer et al. 1987, 1988), a putative mechanism enabling simultaneous sugar utilization was proposed (Fig. 3c) (Kim et al. 2009). Instead of PTS, the sugar transport system of L. brevis consists of $\mathrm{H}^{+}$symporters that operate at the expense of the proton motive force for the translocation of sugar. As shown by the work of Saier (Djordjevic et al. 2001; Ye and Saier 1995a, b), when glucose is present in the media, the transport mechanism for secondary sugar such as xylose or galactose changes from $\mathrm{H}^{+}$symport to facilitated diffusion. This results in xylose or galactose in the cytosol being excreted into medium, thereby achieving inducer exclusion. However, it also opens up the possibility that external (secondary) sugars can be imported via facilitated diffusion, depending on the cytosolic concentration of that sugar. Moreover, introduction of a secondary sugar into cytosol via facilitated diffusion can relieve the transcription repression by the repressor protein. Consequently, the $\mathrm{CcpA}-\mathrm{Hpr}$ complex-mediated transcriptional repression solely regulates the CCR in $L$. brevis. Through this mechanism, the co-presence of glucose and a pentose sugar possibly relaxes the CCR of sugar utilization.

A similar CCR-negative phenotype has been found in two other Lactobacillus species. Lactobacillus buchneri NRRL B-30929, a strain isolated at a fuel ethanol production facility, exhibited co-utilization of glucose and xylose (Liu et al. 2008). Fermentation of lignocellulosic hydrolysate by NRRL B-30929 demonstrated that the strain could simultaneously and completely consume mixed sugars, while producing lactate, acetate, and ethanol (Liu et al. 2009). Recently, we generated a CCR-negative mutant of Lactobacillus pentosus by repeated selection in the presence of 2-deoxyglucose (Kim et al. 2010a). This derived strain can utilize a broad range of sugar substrates simultaneously with glucose. Notably, both $L$. pentosus JH5XP5 and L. buchneri NRRL B-30929 are able to produce ethanol with similar or higher molar yields than lactate when grown on hexoses, suggesting the potential use of these strains in the bio-ethanol production from lignocellulosic biomass.

\section{Other CCR-negative microorganisms}

Although CCR is considered a common regulation mechanism for microorganisms, there are several microbes for which simultaneous utilization of mixed carbon sources has been reported. Many LAB are able to consume citrate with glucose simultaneously (Kennes et al. 1991; Ramos et al. 1994). Streptococcus thermophilus was shown to co-metabolize lactose and sucrose (Poolman et al. 1995). Co-utilization of glucose and glycerol has been observed in Lactobacillus reuteri and L. brevis (El-Ziney et al. 1998; Veiga da Cunha and Foster 1992). Maltose and fructose cofermentation has been reported in L. brevis subsp. lindneri CB1 (Gobbetti and Corsetti 1996). Other bacteria have also been shown to simultaneously consume sugars as well. Clostridium thermohydrosulfuricum can co-metabolize mixtures of glucose and xylose as well as combinations of cellobiose, xylose, and xylobiose (Cook et al. 1993; Slaff and Humphrey 1986).

Ruminal bacteria are able to degrade cellulose and hemicelluloses and to ferment the resulting sugars. Two Butyrivibrio fibrisolvens strains were shown to utilize xylose and glucose simultaneously (Marounek and Kopecny 1994). B. fibrisolvens strain D1 consumed glucose and xylose simultaneously, but preferred xylose over arabinose. Interestingly, this strain showed strong preference for disaccharides such as maltose and cellobiose over glucose and pentose sugars (Strobel and Dawson 1993). Ruminococcus albus preferred cellobiose over xylose and 
arabinose as well, but was able to utilize glucose and xylose simultaneously (Thurston et al. 1994). Another ruminal bacterium, Prevotella ruminicola $\mathrm{B}_{1} 4$, was shown to simultaneously metabolize pentoses and glucose or maltose, but the strain preferentially fermented pentoses over cellobiose and preferred xylose to sucrose (Strobel 1993).

Finally, two eukaryotic strains have shown CCRnegative phenotypes. Maheshwari and Balasubramanyam reported that an unidentified thermophilic fungus could simultaneously consume glucose and sucrose (Maheshwari and Balasubramanyam 1988). In addition Candida shehatae, a strain used for the lignocellulosic biomass utilization, exhibited simultaneous consumption of glucose with xylose (Kastner et al. 1998; Kastner and Roberts 1990).

\section{Conclusion}

In terms of substrate utilization and predictable control of overall process, CCR-negative microorganisms appear ideal for mixed sugar utilization. However, the fermentation characteristics of only a few CCR-negative strains have been studied, much less their potential application for the lignocellulose-based processes. Most current strain development mainly focuses on the product yield, for example, by removing the byproduct formation or enabling xylose utilization. However, when considering the whole production scheme, it is just as important to consider how the fermentation host strains consume the mixed substrates as how much target bioproduct they produce.

Open Access This article is distributed under the terms of the Creative Commons Attribution Noncommercial License which permits any noncommercial use, distribution, and reproduction in any medium, provided the original author(s) and source are credited.

\section{References}

Bertilsson M, Olofsson K, Liden G (2009) Prefermentation improves xylose utilization in simultaneous saccharification and cofermentation of pretreated spruce. Biotechnol Biofuels 2:8

Boerjan W, Ralph J, Baucher M (2003) Lignin biosynthesis. Annu Rev Plant Biol 54:519-546

Bothast RJ, Nichols NN, Dien BS (1999) Fermentations with new recombinant organisms. Biotechnol Progr 15(5):867-875

Buchanan BB, Gruissem W, Jones RL (2000) Biochemistry and molecular biology of plants. American Society of Plant Physiologists, Rockville

Bunch PK, MatJan F, Lee N, Clark DP (1997) The ldhA gene encoding the fermentative lactate dehydrogenase of Escherichia coli. Microbiology 143:187-195

Chaillou S, Bor Y-C, Batt CA, Postma PW, Pouwels PH (1998) Molecular cloning and functional expression in Lactobacillus plantarum 80 of $x y l T$, encoding the $\mathrm{D}$-xylose- $\mathrm{H}^{+}$symporter of
Lactobacillus brevis. Appl Environ Microbiol 64(12):47204728

Chaillou S, Pouwels PH, Postma PW (1999) Transport of D-xylose in Lactobacillus pentosus, Lactobacillus casei, and Lactobacillus plantarum: evidence for a mechanism of facilitated diffusion via the phosphoenolpyruvate: mannose phosphotransferase system. J Bacteriol 181(16):4768-4773

Chaillou S, Postma PW, Pouwels PH (2001) Contribution of the phosphoenolpyruvate:mannose phosphotransferase system to carbon catabolite repression in Lactobacillus pentosus. Microbiology 147(3):671-679

Cook GM, Janssen PH, Morgan HW (1993) Simultaneous uptake and utilization of glucose and xylose by Clostridium thermohydrosulfuricum. FEMS Microbiol Lett 109(1):55-61

Dale BE, Moreira MJ (1982) A freeze explosion technique for increasing cellulose hydrolysis. Biotechnol Bioeng 12:31-43

Djordjevic GM, Tchieu JH, Saier MH (2001) Genes involved in control of galactose uptake in Lactobacillus brevis and reconstitution of the regulatory system in Bacillus subtilis. J Bacteriol 183(10):3224-3236

El-Ziney MG, Arneborg N, Uyttendaele M, Debevere J, Jakobsen M (1998) Characterization of growth and metabolites production of Lactobacillus reuteri during glucose/glycerol cofermentation in batch and continuous cultures. Biotechnol Lett 20(10):913-916

Gobbetti M, Corsetti A (1996) Co-metabolism of citrate and maltose by Lactobacillus brevis subsp. lindneri CB1 citrate-negative strain: effect on growth, end-products and sourdough fermentation. Z Lebensm-Unters Forsch 203(1):82-87

Gosalbes MJ, Monedero V, Perez-Martinez G (1999) Elements involved in catabolite repression and substrate induction of the lactose operon in Lactobacillus casei. J Bacteriol 181(13):39283934

Hahn-Hagerdal B, Karhumaa K, Jeppsson M, Gorwa-Grauslund MF (2007) Metabolic engineering for pentose utilization in Saccharomyces cerevisiae. Biofuels 108:147-177

Ho NW, Chen Z, Brainard AP (1998) Genetically engineered Saccharomyces yeast capable of effective cofermentation of glucose and xylose. Appl Environ Microbiol 64(5):1852-1859

Holtzapple MT, Lundeen JE, Sturgis R, Lewis JE, Dale BE (1992) Pretreatment of lignocellulosic municipal solid waste by ammonia fiber explosion (AFEX). Appl Biochem Biotechnol 34-5:521

Joachimsthal EL, Rogers PL (2000) Characterization of a highproductivity recombinant strain of Zymomonas mobilis for ethanol production from glucose/xylose mixtures. Appl Biochem Biotechnol 84-6:343-356

Kastner JR, Roberts RS (1990) Simultaneous fermentation of D-xylose and glucose by Candida shehatae. Biotechnol Lett 12(1):57-60

Kastner JR, Jones WJ, Roberts RS (1998) Simultaneous utilization of glucose and D-xylose by Candida shehatae in a chemostat. J Ind Microbiol Biotechnol 20(6):339-343

Kennes C, Veiga MC, Dubourguier HC, Touzel JP, Albagnac G, Naveau H, Nyns EJ (1991) Trophic relationships between Saccharomyces cerevisiae and Lactobacillus plantarum and their metabolism of glucose and citrate. Appl Environ Microbiol 57 (4):1046-1051

Kim J-H, Shoemaker SP, Mills DA (2009) Relaxed control of sugar utilization in Lactobacillus brevis. Microbiology 155(4):13511359

Kim J-H, Mills DA, Block DE, Shoemaker SP (2010a) Atypical ethanol production by carbon catabolite derepressed lactobacilli. Bioresour Technol 101(22):8790-8797

Kim J-H, Mills DA, Block DE, Shoemaker SP (2010b) Conversion of rice straw to bio-based chemicals: an integrated process using Lactobacillus brevis. Appl Microbiol Biotechnol 86(5):13751385 
Kimata K, Takahashi H, Inada T, Postma P, Aiba H (1997) cAMP receptor protein-cAMP plays a crucial role in glucoselactose diauxie by activating the major glucose transporter gene in Escherichia coli. Proc Natl Acad Sci USA 94(24):1291412919

Kuyper M, Winkler AA, van Dijken JP, Pronk JT (2004) Minimal metabolic engineering of Saccharomyces cerevisiae for efficient anaerobic xylose fermentation: a proof of principle. FEMS Yeast Res 4(6):655-664

Lau MW, Dale BE (2009) Cellulosic ethanol production from AFEXtreated corn stover using Saccharomyces cerevisiae 424A(LNHST). Proc Natl Acad Sci USA 106(5):1368-1373

Lawford HG, Rousseau JD (2002) Performance testing of Zymomonas mobilis metabolically engineered for cofermentation of glucose, xylose, and arabinose. Appl Biochem Biotechnol 98:429-448

Leandro MJ, Fonseca C, Goncalves P (2009) Hexose and pentose transport in ascomycetous yeasts: an overview. FEMS Yeast Res 9(4):511-525

Lee WJ, Kim MD, Ryu YW, Bisson LF, Seo JH (2002) Kinetic studies on glucose and xylose transport in Saccharomyces cerevisiae. Appl Microbiol Biotechnol 60(1-2):186-191

Lee WJ, Kim MD, Yoo MS, Ryu YW, Seo JH (2003) Effects of xylose reductase activity on xylitol production in two-substrate fermentation of recombinant Saccharomyces cerevisiae. J Microbiol Biotechnol 13(5):725-730

Lengeler JW (1996) The phosphoenolpyruvate-dependent carbohydrate: phosphotransferase system (PTS) and control of carbon source utilization. In: Lin ECC, Lynch AS (eds) Regulation of gene expression in Escherichia coli. R. G. Landes Co., Austin, Texas, pp 231-254

Lengeler JW, Drews G, Schlegel HG (1999) Biology of the prokaryotes. Blackwell, New York

Liu SQ, Skinner-Nemec KA, Leathers TD (2008) Lactobacillus buchneri strain NRRL B-30929 converts a concentrated mixture of xylose and glucose into ethanol and other products. J Ind Microbiol Biotechnol 35(2):Cp4-Cp81

Liu S, Bischoff KM, Hughes SR, Leathers TD, Price NP, Qureshi N, Rich JO (2009) Conversion of biomass hydrolysates and other substrates to ethanol and other chemicals by Lactobacillus buchneri. Lett Appl Microbiol 48(3):337-342

Maheshwari R, Balasubramanyam PV (1988) Simultaneous utilization of glucose and sucrose by thermophilic fungi. J Bacteriol 170 (7):3274-3280

Mahr K, Hillen W, Titgemeyer F (2000) Carbon catabolite repression in Lactobacillus pentosus: analysis of the ccpA region. Appl Environ Microbiol 66(1):277-283

Marasco R, Muscariello L, Varcamonti M, De Felice M, Sacco M (1998) Expression of the bglH gene of Lactobacillus plantarum is controlled by carbon catabolite repression. J Bacteriol 180 (13):3400-3404

Marounek M, Kopecny J (1994) Utilization of glucose and xylose in ruminal strains of Butyrivibrio fibrisolvens. Appl Environ Microbiol 60(2):738-739

Meinander NQ, HahnHagerdal B (1997) Influence of cosubstrate concentration on xylose conversion by recombinant, XYL1expressing Saccharomyces cerevisiae: a comparison of different sugars and ethanol as cosubstrates. Appl Environ Microbiol 63 (5):1959-1964

Meinander NQ, Boels I, Hahn-Hagerdal B (1999) Fermentation of xylose/glucose mixtures by metabolically engineered Saccharomyces cerevisiae strains expressing XYL1 and XYL2 from Pichia stipitis with and without overexpression of TAL1. Bioresour Technol 68(1):79-87

Morel F, Frot-Coutaz J, Aubel D, Portalier R, Atlan D (1999) Characterization of a prolidase from Lactobacillus delbrueckii subsp. bulgaricus CNRZ 397 with an unusual regulation of biosynthesis. Microbiology 145(2):437-446

Nichols NN, Dien BS, Bothast RJ (2001) Use of catabolite repression mutants for fermentation of sugar mixtures to ethanol. Appl Microbiol Biotechnol 56(1-2):120-125

Olofsson K, Wiman M, Liden G (2010) Controlled feeding of cellulases improves conversion of xylose in simultaneous saccharification and co-fermentation for bioethanol production. J Biotechnol 145(2):168-175

Parker C, Barnell WO, Snoep JL, Ingram LO, Conway T (1995) Characterization of the Zymomonas mobilis glucose facilitator gene product $(\mathrm{Glf})$ in recombinant Escherichia coli-examination of transport mechanism, kinetics and the role of glucokinase in glucose transport. Mol Microbiol 15(5):795-802

Pitkanen JP, Aristidou A, Salusjarvi L, Ruohonen L, Penttila M (2003) Metabolic flux analysis of xylose metabolism in recombinant Saccharomyces cerevisiae using continuous culture. Metab Eng 5 (1): $16-31$

Poolman B, Knol J, Mollet B, Nieuwenhuis B (1995) Regulation of bacterial sugar- $\mathrm{H}^{+}$symport by phosphoenolpyruvate-dependent enzyme I/HPr-mediated phosphorylation. Proc Natl Acad Sci USA 92(3):778-782

Ramos A, Jordan KN, Cogan TM, Santos H (1994) ${ }^{13} \mathrm{C}$ nuclear magnetic resonance studies of citrate and glucose cometabolism by Lactococcus lactis. Appl Environ Microbiol 60(6):1739-1748

Reizer J, Peterkofsky A, Romano AH (1987) Evidence for Hpr in Lactobacillus brevis in the absence of transport function. Abstr Annu Meet Am Soc Microbiol 87:228

Reizer J, Peterkofsky A, Romano AH (1988) Evidence for the presence of heat-stable protein (HPr) and ATP-dependent HPr kinase in heterofermentative lactobacilli lacking phosphoenolpyruvate:glycose phosphotransferase activity. Proc Natl Acad Sci USA 85(7):2041-2045

Ropars M, Marchal R, Pourquie J, Vandecasteele JP (1992) Large scale enzymatic hydrolysis of agricultural lignocellulosic biomass. Part 1: pretreatment procedures. Bioresour Technol 42 (3): 197-204

Saha BC (2003) Hemicellulose bioconversion. J Ind Microbiol Biotechnol 30(5):279-291

Schell DJ, Torget R, Power A, Walter PJ, Grohmann K, Hinman ND (1991) A technical and economic analysis of acid catalyzed steam explosion and dilute sulfuric acid pretreatments using wheat straw or aspen wood chips. Appl Biochem Biotechnol 289:87-97

Schick J, Weber B, Klein JR, Henrich B (1999) PepR1, a CcpA-like transcription regulator of Lactobacillus delbrueckii subsp lactis. Microbiology 145(11):3147-3154

Sedlak M, Ho NWY (2004a) Characterization of the effectiveness of hexose transporters for transporting xylose during glucose and xylose co-fermentation by a recombinant Saccharomyces yeast. Yeast 21(8):671-684

Sedlak M, Ho NWY (2004b) Production of ethanol from cellulosic biomass hydrolysates using genetically engineered Saccharomyces yeast capable of cofermenting glucose and xylose. Appl Biochem Biotechnol 113-16:403-416

Slaff GF, Humphrey AE (1986) The growth of Clostridium thermydrosulfuricum on multiple substrates. Chem Eng Commun 45(16):33-51

Strobel HJ (1993) Pentose utilization and transport by the ruminal bacterium Prevotella ruminicola. Arch Microbiol 159(5):465471

Strobel HJ, Dawson KA (1993) Xylose and arabinose utilization by the rumen bacterium Butyrivibrio fibrisolvens. FEMS Microbiol Lett 113(3):291-296

Stulke J, Hillen W (1999) Carbon catabolite repression in bacteria. Curr Opin Microbiol 2(2):195-201 
Stulke J, Hillen W (2000) Regulation of carbon catabolism in Bacillus species. Annu Rev Microbiol 54(5):849-880

Thurston B, Dawson KA, Strobel HJ (1994) Pentose utilization by the ruminal bacterium Ruminococcus albus. Appl Environ Microbiol 60(4):1087-1092

Titgemeyer F, Hillen W (2002) Global control of sugar metabolism: a Gram-positive solution. Anton Leeuw Int J G 82(1-4):59-71

Veiga da Cunha M, Foster MA (1992) Sugar-glycerol cofermentations in lactobacilli: the fate of lactate. J Bacteriol 174(3):1013-1019

Veyrat A, Monedero V, Perez-Martinez G (1994) Glucose transport by the phosphoenolpyruvate:mannose phosphotransferase system in Lactobacillus casei ATCC 393 and its role in carbon catabolite repression. Microbiology 140(5):1141-1149

Viana R, Monedero V, Dossonnet V, Vadeboncoeur C, Perez-Martinez G, Deutscher J (2000) Enzyme I and HPr from Lactobacillus casei: their role in sugar transport, carbon catabolite repression and inducer exclusion. Mol Microbiol 36(3):570-584
Warner JB, Lolkema JS (2003) CcpA-dependent carbon catabolite repression in bacteria. Microbiol Mol Biol Rev 67(4):475

Ye JJ, Saier MH (1995a) Allosteric regulation of the glucose: $\mathrm{H}^{+}$ symporter of Lactobacillus brevis: cooperative binding of glucose and $\mathrm{HPr}($ ser-P). J Bacteriol 177(7):1900-1902

Ye JJ, Saier MH (1995b) Cooperative binding of lactose and the phosphorylated phosphocarrier protein $\operatorname{HPr}(\mathrm{Ser}-\mathrm{P})$ to the lactose/ $\mathrm{H}^{+}$symport permease of Lactobacillus brevis. Proc Natl Acad Sci USA 92(2):417-421

Zhang M, Eddy C, Deanda K, Finkelstein M, Picataggio S (1995) Metabolic engineering of a pentose metabolism pathway in ethanologenic Zymomonas mobilis. Science 267(5195):240243

Zuniga M, Champomier-Verges M, Zagorec M, Perez-Martinez G (1998) Structural and functional analysis of the gene cluster encoding the enzymes of the arginine deiminase pathway of Lactobacillus sake. J Bacteriol 180(16):4154-4159 